\title{
An Independent Three-Dimensional (3D) Dose Verification System for Elekta Unity MR-Linac Online Plans
}

\section{Danny Lee ( Danny.Lee@AHN.ORG )}

Allegheny Health Network https://orcid.org/0000-0003-3270-1066

\section{Daniel Pavord}

Allegheny Health Network

\section{Seungjong Oh}

Allegheny Health Network

Jason Sohn

Allegheny Health Network

\section{Research Article}

Keywords: Online adaptive radiotherapy, 3D dose verification, MR-linac, IMRT QA, online quality assurance

Posted Date: April 14th, 2021

DOl: https://doi.org/10.21203/rs.3.rs-381411/v1

License: (9) This work is licensed under a Creative Commons Attribution 4.0 International License. Read Full License 


\section{Abstract}

\section{Purpose}

To implement an independent 3D dose verification system with RayStation (RaySearch, Stockholm, Sweden) for online adaptive radiotherapy on Elekta Unity MR-Linac (MRL).

\section{Methods}

Plan quality of simple-single-field and intensity-modulated radiotherapy (IMRT) plans were investigated in a comparison of (1) Monte-Carlo calculated data using MRL Monaco with high magnetic field (1.5 T) and (2) Collapsed-Cone calculated data using RayStation. The dose quality of RayStation plans, compared to corresponding Monaco plans, was (1) visually inspected in percentage depth-dose curves, inline and crossline profiles, and (2) quantified in 3D gamma-passing-rates. Processing time was measured to evaluate the practical efficacy of our system using 5 prostate IMRT plans.

\section{Results}

Compared to Monaco simple-single-field plans as ground truth, RayStation simple-single-field plans achieved an average $95.7 \%$ and $98.5 \%$ in $2 \% / 2 \mathrm{~mm}$ and $3 \% / 3 \mathrm{~mm}$ of $3 \mathrm{D}$ gamma criteria, respectively. Gantry angle dependency in simple-single-field plans was $<2 \%$ in both $2 \% / 2 \mathrm{~mm}$ and $3 \% / 3 \mathrm{~mm}$, and field size dependency was $<5 \%$ in $2 \% / 2 \mathrm{~mm}$ and $<2 \%$ in $3 \% / 3 \mathrm{~mm}$. Compared to Monaco IMRT plans, RayStation IMRT plans achieved an average $95.1 \%(3 \% / 3 \mathrm{~mm})$. The entire processing time of the independent 3D dose verification system was an average approximately $200 \mathrm{~s}$.

\section{Conclusions}

This was the study to implement an independent 3D dose verification process using RayStation with an in-house 3D gamma analysis software. This led to an average $95 \%$ plan quality in $3 \% / 3 \mathrm{~mm}$ gamma criteria and added an average $200 \mathrm{~s}$ throughout the entire verification processes. These results demonstrate that this approach can be applicable and efficient for online quality assurance for MRL online adaptive radiotherapy planning.

\section{Introduction}

Patient specific pre-treatment quality assurance (QA) is essential to ensure the plan quality of individual treatment plans. ${ }^{1,2}$ Various QA devices are utilized to verify clinical acceptability for intensity-modulated plans. ${ }^{3-5}$ However, these QA devices are insufficient for online adaptive radiotherapy due to that it requires online QA(s) during day-to-day plan adaptations. ${ }^{6}$ To overcome this issue, an independent dose verification system is required to confirm plan quality without interrupting online adaptive radiotherapy.

To perform dose verification for an Elekta Unity (Elekta AB, Stockholm, Sweden) MRI-Linac (MRL) equipped with a 1.5 T MRI scanner (Philips Healthcare) during online adaptive radiotherapy, Chen, et al., 
developed an in-house software tool to account for the influence of the high magnetic field. ${ }^{7}$ They considered transverse magnetic field across several depth doses and various profiles in dose calculation. In addition, a commercial software package (RadCalc v6.3, LifeLine Software, Inc.) was evaluated for the Elekta Unity MRI-Linac (Elekta AB, Stockholm, Sweden) to verify plan quality prior to patient treatment. ${ }^{8}$ Beam profiles affected by a $1.5 \mathrm{~T}$ magnetic field at gantry $0^{\circ}$ and $270^{\circ}$ for the field sizes of various simple single fields (i.e., square and rectangular shapes) at several depths were utilized to build a relative beam model on the RadCalc.

The third party treatment planning systems (TPS) were utilized to build individual MRL beam models using asymmetry dose profiles calculated on a Monte Carlo algorithm on Monaco v5.4 (Elekta AB, Stockholm, Sweden) due to the effect of a $1.5 \mathrm{~T}$ magnetic field. Goodwin, et al. ${ }^{9}$ and Li, et al., ${ }^{10}$ were utilized RayStation TPS(s) to build a MRL beam model and commission the MRL beam model for developing RayStation plans. Then, the plan quality of MRL Monaco plans, compared to corresponding RayStation plans was verified by visually inspecting dose difference ${ }^{9}$ and quantifying 3D gamma pass rates at an average $90 \%$ in $3 \% / 3 \mathrm{~mm}$ gamma criteria. ${ }^{10}$ In addition, the Pinnacle (Philips, Best, the Netherlands) TPS was used to develop quasi MRL plans incorporated MRL characteristics and compare with MRL plans developed on Monaco v5.4. ${ }^{11}$

In overall, online adaptive radiotherapy requires an independent dose calculation system for the secondary dose verification on MRL. However, it has dependency on the characteristics of individual MRL(s) and TPS(s), and commercial software currently provides a comparison of a single point dose ${ }^{8}$ and the third party TPS also provides an average $90 \%$ of plan quality in 3D doses. Hence, this study aims to implement an independent and 3D dose verification system with RayStation for online QA during online adaptive radiotherapy planning. We devised in-house gamma analysis software, which can compare two plans, and provides 3D volumetric gamma analysis.

\section{Methods}

An independent 3D dose verification system using RayStation (Version 9A) is comprised of five steps: (1) developing MRL plans on an MRL Monaco (Version 5.40.01), (2) exporting Monaco DICOM data to the pre-determined folder, (3) importing the Monaco DICOM data to RayStation and dose calculation, (4) exporting RayStation DICOM data (only plan and dose) to the same pre-determined folder, and (5) comparing Monaco and RayStation plan doses using an in-house 3D gamma analysis software. Figure 1 shows the workflow of an independent 3D dose verification system in five steps.

\subsection{Developing MRL Monaco plans}

Various simple single field plans (i.e., simple 3D plans with a single square and rectangular field) were made using MRL Monaco with Monte Carlo® algorithm. Those plans were calculated using GPUMCD (GPU-oriented Monte Carlo dose calculation algorithm) in MRL Monaco TPS with high magnetic field (1.5 T) and a 7MV FFF (flattening filter free) energy. The simple single field plans with 100 monitor units (MU) 
at SSD $133.5 \mathrm{~cm}$ across various field sizes $\left(3 \times 3 \mathrm{~cm}^{2}, 5 \times 5 \mathrm{~cm}^{2}, 10 \times 10 \mathrm{~cm}^{2}, 10 \times 10 \mathrm{~cm}^{2}, 15 \times 15 \mathrm{~cm}^{2}\right.$, $20 \times 20 \mathrm{~cm}^{2}, 22 \times 22 \mathrm{~cm}^{2}$ and $\left.40 \times 22 \mathrm{~cm}^{2}\right)$ and gantry angles $\left(0^{\circ}, 90^{\circ}, 180^{\circ}\right.$ and $\left.270^{\circ}\right)$ were developed using a uniform water phantom and an MRL couch. The MR coil was absent during the development of simple single field plans. A $0.3 \mathrm{~cm}$ grid spacing and a $0.5 \%$ statistical uncertainty were used during dose calculation for all simple single field plans. These Monaco simple single field plans were used as inputs of a beam model built on RayStation and following the beam model verification whilst comparing to RayStation simple single field plans (see the Sect. 2.2).

In addition, 7-field intensity-modulated radiation therapy (IMRT) plans were developed on MRL Monaco using volumetric modulated arc therapy (VMAT) plans of five prostate cancer patients received external beam radiotherapy. The CT images and structures of VMAT plans were manually exported from Monaco v5.11.02 (Elekta AB, Stockholm, Sweden) as a DICOM format and imported to MRL Monaco. The same CT images, structures and prescriptions (7000 cGy with 28 fractions for a plan and $4600 \mathrm{cGy}$ with 23 fractions for 4 plans) were utilized during the plan development of 7-field IMRT plans. For the 7-field IMRT plans, a MV 7FFF beam was used with MRL plan parameters (i.e., Gantry angles at every $51^{\circ}$, collimator $0^{\circ}$, the isocenter location at the center of planning target volume, dose rates at $425 \mathrm{MU} / \mathrm{min}$, and MR coil and MR couch components). A $0.3 \mathrm{~cm}$ grid spacing and a $0.5 \%$ statistical uncertainty were used during dose calculation for all 7-field IMRT plans.

\subsection{Developing MRL RayStation plans}

Prior to developing MRL RayStation plans, an MRL beam model was built on RayStation using inline and crossline profiles, and percentage depth dose (PDD) curves of the Monaco simple single field plans which have a zero gantry angle. The PDD curves and profiles were extracted from multiple depth doses at maximum dose, $5 \mathrm{~cm}, 10 \mathrm{~cm}$ and $20 \mathrm{~cm}$ as inputs utilized during RayStation beam modeling. Profiles and offsets of an MRL RayStation beam were adjusted for best matching to the corresponding the input profiles across all fields and depths. RayStation simple single field plans were then developed using the new MRL RayStation beam and a Collapsed Cone algorithm to calculate plan doses. The same uniform water phantom and MRL couch were used in the development of Monaco simple single field plans. The same grid spacing and statistical uncertainty of Monaco plans were used during dose calculation for all simple single field plans. We intended to isolate the source of discrepancy possibly occurred in beam commissioning process before proceeding to complex IMRT plan comparison.

In addition, a DICOM importing tool was developed using Python scripts in RayStation to automatically modify DICOM headers, accounting for the difference of DICOM properties between Monaco and RayStation. It copied DICOM properties which are absent such as SoftwareVersion and FrameOfReferenceUID from a reference CT image to a radiotherapy (RT) Plan. An essential DICOM properties such as an $x$ MLC component was additionally added to BeamLimitingDeviceSequence due to the absence of the $x$ MLC component in the MRL Monaco plan. During importing MRL Monaco plans, the tool automatically allocated a CT scanner and a treatment machine by matching their identical names to one of the pre-registered CT scanners and treatment machines. 
To develop MRL RayStation plans, our scripts allowed streamlining the process, so that the MRL Monaco plan, which was presently imported without the modification of plan parameters, was utilized to calculate plan dose using the Collapsed Cone algorithm. The same grid spacing and statistical uncertainty of Monaco plans was used during dose calculation for all 7-field IMRT plans. The grid spacing was manually set in this study after completing our scripts.

\subsection{Dose comparisons and statistical analysis}

RayStation simple single field plans were compared to corresponding Monaco simple single field plans. For dose comparisons between RayStation and Monaco simple single field plans, our in-house gamma analysis software imported and used plan and dose files, and analyzed 3D gamma passing rates. ${ }^{12}$ Gamma analysis with $3 \% / 3 \mathrm{~mm}$ and $2 \% / 2 \mathrm{~mm}$ was performed and 3D gamma passing rates were quantified to compare dose similarity between RayStation and Monaco plans. The gamma passing rates of the simple single field plans were compared to assess the dependency of gantry angles at $0^{\circ}, 90^{\circ}$, $180^{\circ}$ and $270^{\circ}$. Similarly, RayStation 7-field IMRT plans were compared to corresponding Monaco 7-field IMRT plans. For dose comparisons between RayStation and Monaco 7-field IMRT plans, our in-house gamma analysis software was used to perform gamma analysis with $3 \% / 3 \mathrm{~mm}$ gamma criteria.

Using Monaco 7-field IMRT plans, the performance of the proposed independent 3D dose verification system was assessed by measuring the processing time of (1) exporting DICOM data from MRL Monaco, (2) importing DICOM data to RayStation via scripting, (3) calculating plan dose using Collapsed Cone algorithm, (4) exporting DICOM data from RayStation and (5) comparing plan dose using the in-house 3D gamma analysis software.

\section{Results}

In this study, an independent 3D dose verification system was successfully developed by (1) building a new MRL beam model for developing simple single field plans and 7-field IMRT plans on RayStation, (2) implementing Python scripts for importing MRL Monaco plans to RayStation and (3) implementing an inhouse software for gamma analysis.

\subsection{Dose comparison between simple single field plans}

MRL Monaco simple single field plans were compared to corresponding MRL RayStation simple single field plans by using PDD curves and dose profiles, and quantifying 3D dose differences in gamma analysis. A PDD curve, inline (superior to inferior) and crossline (left to right) dose profiles extracted from each 3D dose at the central axis and they were visually inspected. Figure 2 shows an example of a visual inspection from a simple single field plan with a $10 \times 10 \mathrm{~cm}^{2}$ field size and a $10 \mathrm{~cm}$ depth. Both doses of MRL Monaco simple single field plans using Monte Carlo and RayStation simple single field plans using collapsed cone algorithm were normalized to $100 \mathrm{cGy}$ at a $10 \mathrm{~cm}$ depth. 
Both simple single field plans were well agreed in the PDD curve, inline and crossline profiles. The maximum dose of both Monaco and RayStation simple single field plans in the PDD curve (see Fig. 2(a)) was about $146 \mathrm{cGy}$ at a $1.5 \mathrm{~cm}$ depth. Inline and crossline profiles (see Fig. 2(b) and (c)) of both Monaco and RayStation simple single field plans were very similar.

The quality of simple single field plans measured by comparing 3D doses using the in-house gamma analysis software is shown in Fig. 3.

The RayStation simple single field plans with the new MRL beam model showed promising results with an average $95.7 \%$ and $98.5 \%$ in $2 \% / 2 \mathrm{~mm}$ and $3 \% / 3 \mathrm{~mm}$ gamma criteria (see Fig. $3(\mathrm{a})$ and (b)),

respectively. With the same gantry angle, the field size dependency of RayStation simple single field plans in $2 \% / 2 \mathrm{~mm}$ gamma criteria was $3.6 \%$ at $0^{\circ}, 4.6 \%$ at $90^{\circ}, 4.0 \%$ at $180^{\circ}$ and $6.0 \%$ at $270^{\circ}$ but it was $<3.2 \%$ for all gantry angles when excluded the $3 \mathrm{~cm} \times 3 \mathrm{~cm}$ field. In $3 \% / 3 \mathrm{~mm}$ gamma criteria, it was $\leq 1.9 \%$ for all field sizes.

With the same field size, the gantry angle dependency of simple single field plans in $2 \% / 2 \mathrm{~mm}$ gamma criteria was $2.2 \%$ at $3 \mathrm{~cm} \times 3 \mathrm{~cm}, 2.5 \%$ at $5 \mathrm{~cm} \times 5 \mathrm{~cm}, 2.8 \%$ at $10 \mathrm{~cm} \times 10 \mathrm{~cm}, 3.5 \%$ at $20 \mathrm{~cm} \times 20 \mathrm{~cm}$ and $3.0 \%$ at $22 \mathrm{~cm} \times 22 \mathrm{~cm}$. In $3 \% / 3 \mathrm{~mm}$ gamma criteria, it was $<2.2 \%$ for all gantry angles. The gamma passing rate of the same field size (see Fig. $3(\mathrm{~b})$ ) was the smallest at gantry angle $180^{\circ}$ due to the attenuation of a posterior MR receiver coil (i.e., about $0.9 \%$ attenuation) and it was very similar at both gantry angle $90^{\circ}$ and $270^{\circ}$.

\subsection{Dose comparison between 7-field IMRT plans}

MRL Monaco 7-field IMRT plans were compared to corresponding MRL RayStation 7-field IMRT plans by using dose profiles and quantifying 3D dose differences in gamma analysis. Inline and crossline dose profiles extracted from each 3D dose at the central axis and they were visually inspected. Figure 4 shows an example of a visual inspection from a 7-field IMRT plan.

Both Monaco and RayStation 7-field IMRT plans were reasonably well agreed in the longitudinal, inline and crossline profiles. The doses of the Monaco and RayStation plans at the central axis were $4.4 \%$ higher (261 cGy) and - 1.3\% lower (247 cGy) from 250 cGy, respectively.

Figure 5 shows an example of the quality of 7-field IMRT plans (7000 cGy in 28 fractions) which comparing 3D doses using the in-house gamma analysis software. The Monaco 7-field IMRT plan and dose were displayed on RayStation to directly compare to RayStation 7-field IMRT plan and dose.

Both Monaco and RayStation 7-field IMRT plan doses are visually very similar and the target (a solid blue line in Fig. 5(a) and (b)) entirely remains in yellow and orange colors in the range between $7271 \mathrm{cGy}$ (95\% of 7654 cGy) and 6889 cGy ( $90 \%$ of 7654 cGy), respectively. Their dose difference in gamma remains in less than 3.0\% which the red color (see Fig. 5(c)) was minimal except for the entry of beams. 


\subsection{Performance assessment of the independent dose verification system}

An independent 3D dose verification system using Monaco 7-field IMRT plans was assessed by measuring the processing time of individual steps as a function of its performance. An average of the total processing time was approximately $200 \mathrm{~s}$ which summed individual processing time of (1) DICOM export from MR-Linac Monaco (34 s), (2) DICOM import to RayStation via scripting (80 s), (3) dose calculation (24 s), (4) DICOM export from RayStation (26 s), and (5) 3D Gamma analysis using the inhouse gamma analysis software (36 s).

In addition, an average of gamma passing rates from five 7-field IMRT plans was $95.1 \%$ in $3 \% / 3 \mathrm{~mm}$ gamma criteria and individual gamma passing rates were $96.3 \%, 93.5,94.7 \%, 95.9 \%$ and $95.1 \%$, respectively.

\section{Discussion}

Online adaptive radiotherapy using MRL requires an independent dose verification to confirm plan quality in a short period time in the presence of patients on the treatment couch. This study implemented an independent 3D dose verification system using RayStation and in-house 3D gamma analysis software. Using the independent 3D dose verification system, we demonstrated (1) a superior plan quality ( $<98.5 \%$ in $3 \% / 3 \mathrm{~mm}$ gamma criteria) of RayStation simple single field and 7-field IMRT plans whilst comparing to corresponding Monaco simple single field and 7-field IMRT plans, and (2) its applicability for independently verifying plan quality during MRL online adaptive radiotherapy.

An independent 3D dose verification needs to account for a $1.5 \mathrm{~T}$ high magnetic field ${ }^{7-9}$ so this study also utilized Monte Carlo ${ }^{\circledR}$ calculated data (i.e., Asymmetry profile data) during beam modeling on RayStation. In the use of the beam model, RayStation simple single field plans compared to corresponding Monaco simple single field plans achieved an average $95.7 \%$ and $98.7 \%$ of plan quality in $2 \% / 2 \mathrm{~mm}$ and $3 \% / 3 \mathrm{~mm}$ gamma criteria (see Fig. 3), respectively. In addition, RayStation 7-field IMRT plans achieved an average $95.1 \%$ in $3 \% / 3 \mathrm{~mm}$ gamma criteria, which can be (1) acceptable to minimize dependency of individual MRL(s) and (2) applicable to verify plan quality during the online QA(s) of dayto-day plan adaptations. ${ }^{6}$

Speed can be essential to minimize the variability of organ locations and changing the position of patient setups during dose verification and also efficiency can be very important to improve the performance of an independent 3D dose verification system. This study implemented Python scripts for importing Monaco DICOM data to RayStation and an in-house 3D gamma analysis software for comparing plan quality, resulted in that entire dose verification can be achievable in approximately $200 \mathrm{~s}$. In addition, automated processes reduced speed and improve efficiency whilst minimizing the involvement of manual processes (i.e., only DICOM export to a pre-determined folder). 
One of the limitations of the present study was the dependency of gantry angles (see Fig. 3). The

accuracy of dose calculation can be determined by precise beam modeling on individual TPS(s) ${ }^{7-11}$ and thus our beam model was built on RayStation using the characteristic and specific information of MRL. ${ }^{13,14}$ However, it still included about $2.0 \%$ of gantry angle dependency (see Fig. $3(b)$ ) in $3 \% / 3 \mathrm{~mm}$ gamma criteria, requiring a compensation of gantry angle dependency. ${ }^{9}$

We implemented the in-house software only for comparing 3D dose to analyze plan quality in gamma criteria and also this study did not consider more complicate plans. For better and powerful analysis of plan quality, the dose volume histogram and dose coverage of target and organ at risks could be utilized during online QA and reviewing adaptive plans prior to daily treatment delivery.

\section{Conclusion}

This was the study to implement an independent 3D dose verification system using RayStation with scripts and an in-house 3D gamma analysis software. This led to an average $95 \%$ of plan quality in $3 \% / 3 \mathrm{~mm}$ gamma criteria and added an average $200 \mathrm{~s}$ throughout the entire verification process in the independent 3D dose verification system. These results demonstrate that this approach can be applicable and efficient for online QA during online adaptive radiotherapy with minimal time extension. Further investigations will need to include more heterogeneous target and organ at risk samples.

\section{Declarations}

ACKNOWLEDGEMENTS: The authors thank Scott Hartzell (Senior Physics Specialist, RaySearch) for technical support.

Compliance with Ethical Standards: This study was approved by IRB (Institutional Review Board).

Conflict of interest: The authors have no relevant conflicts of interest to disclose.

\section{References}

1. Kim J, Park S-Y, Kim H, Kim J, Ye S-J, Park J. The sensitivity of gamma-index method to the positioning errors of high-definition MLC in patient-specific VMAT QA for SBRT. Radiat Oncol. 2014;9(1):167. doi:10.1186/1748-717X-9-167

2. Agazaryan N, Solberg TD, DeMarco JJ. Patient specific quality assurance for the delivery of intensity modulated radiotherapy. Journal of Applied Clinical Medical Physics. 2003;4(1):40-50. doi:10.1120/jacmp.v4i1.2540

3. Li G, Zhang Y, Jiang X, et al. Evaluation of the ArcCHECK QA system for IMRT and VMAT verification. Physica Medica. 2013;29(3):295-303. doi:10.1016/j.ejmp.2012.04.005

4. de Vries JHW, Seravalli E, Houweling AC, et al. Characterization of a prototype MR-compatible Delta4 QA system in a 1.5 tesla MR-linac. Phys Med Biol. 2018;63(2):02NT02. doi:10.1088/1361- 
$6560 /$ aa9d26

5. Alhazmi A, Gianoli C, Neppl S, et al. A novel approach to EPID-based 3D volumetric dosimetry for IMRT and VMAT QA. Phys Med Biol. 2018;63(11):115002. doi:10.1088/1361-6560/aac1a6

6. Winkel D, Bol GH, Kroon PS, et al. Adaptive radiotherapy: The Elekta Unity MR-linac concept. Clinical and Translational Radiation Oncology. 2019;18:54-59. doi:10.1016/j.ctro.2019.04.001

7. Chen G-P, Ahunbay E, Li XA. Technical Note: Development and performance of a software tool for quality assurance of online replanning with a conventional Linac or MR-Linac: QA tool for online replanning with MR-Linac. Med Phys. 2016;43(4):1713-1719. doi:10.1118/1.4943795

8. Graves SA, Snyder JE, Boczkowski A, et al. Commissioning and performance evaluation of RadCalc for the Elekta unity MRI-linac. J Appl Clin Med Phys. 2019;20(12):54-62. doi:10.1002/acm2.12760

9. Goodwin E, Nill S, Oelfke U. Commissioning an Independent Dose Calculation System for the Unity MR-Linac. Radiotherapy and Oncology. 2019;133:S978-S979. doi:10.1016/S0167-8140(19)32226-1

10. Li Y, Wang B, Ding S, et al. Feasibility of using a commercial collapsed cone dose engine for 1.5T MR-LINAC online independent dose verification. Physica Medica. 2020;80:288-296.

doi:10.1016/j.ejmp.2020.11.014

11. van de Schoot AJAJ, van den Wollenberg W, Carbaat C, et al. Evaluation of plan quality in radiotherapy planning with an MR-linac. Physics and Imaging in Radiation Oncology. 2019;10:19-24. doi:10.1016/j.phro.2019.04.004

12. Park JM, Kim J, Park S-Y, Oh DH, Kim S-T. Reliability of the gamma index analysis as a verification method of volumetric modulated arc therapy plans. Radiat Oncol. 2018;13(1):175. doi:10.1186/s13014-018-1123-x

13. Snyder JE, St-Aubin J, Yaddanapudi S, et al. Commissioning of a 1.5T Elekta Unity MR-linac: A single institution experience. J App/ Clin Med Phys. 2020;21(7):160-172. doi:10.1002/acm2.12902

14. Tijssen RHN, Philippens MEP, Paulson ES, et al. MRI commissioning of 1.5T MR-linac systems - a multi-institutional study. Radiotherapy and Oncology. 2019;132:114-120. doi:10.1016/j.radonc.2018.12.011

\section{Figures}


(a) MRL Monaco plan
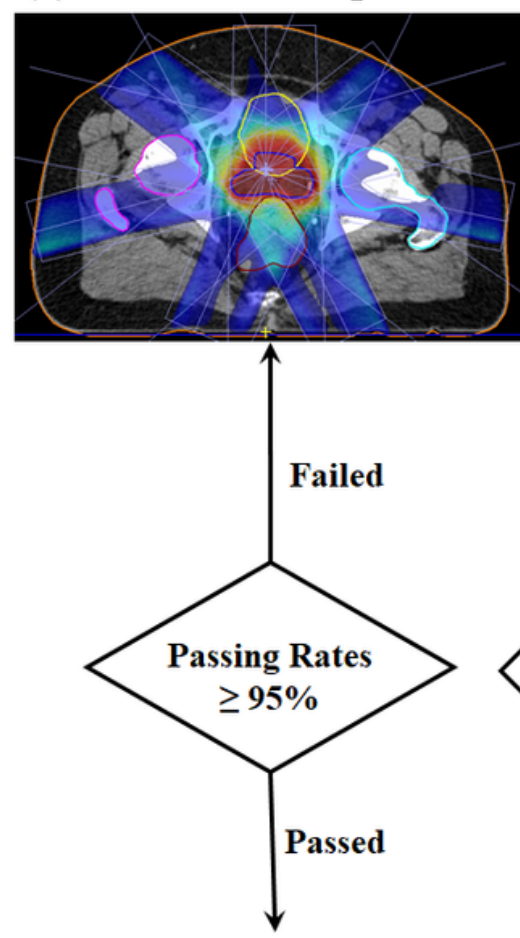

(b) Monaco DICOM data

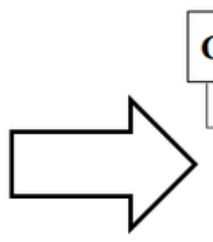

\section{CT/MR images}

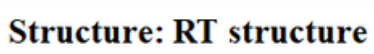

Plan: Monaco RT plan

Dose: Monaco RT dose

(e) 3D dose comparison

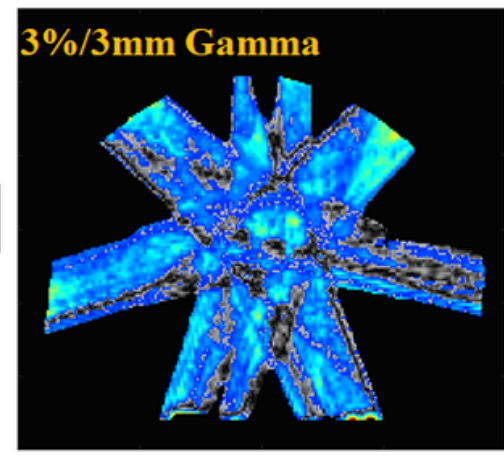

(c) MRL RayStation plan
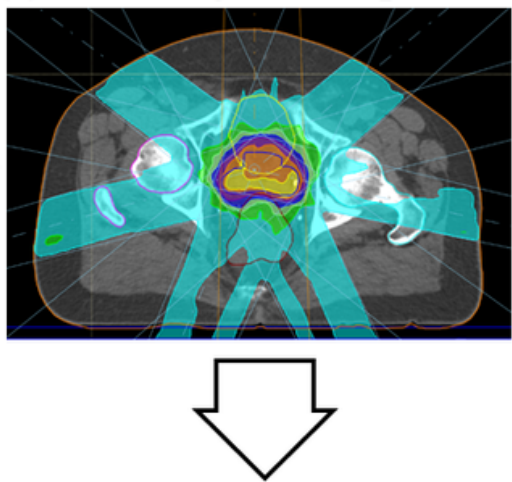

(d) RayStation DICOM data

\section{Figure 1}

The workflow of an independent 3D dose verification system. (a) Developing MRL Monaco plans, (b) exporting Monaco DICOM data to a pre-determined folder, (c) importing the Monaco DICOM data to RayStation and dose calculation, (d) exporting RayStation DICOM data (only plan and dose) to the same pre-determined folder, and (d) comparing Monaco and RayStation plan doses using an in-house 3D gamma analysis software.

(a) PDD in cGy

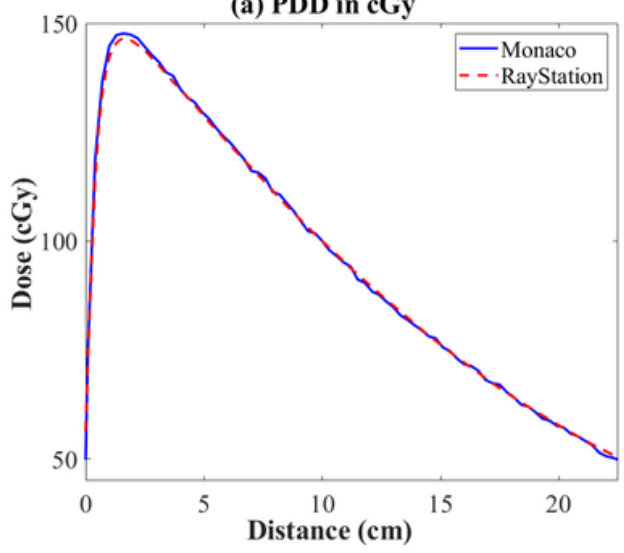

(b) Inline profile in cGy

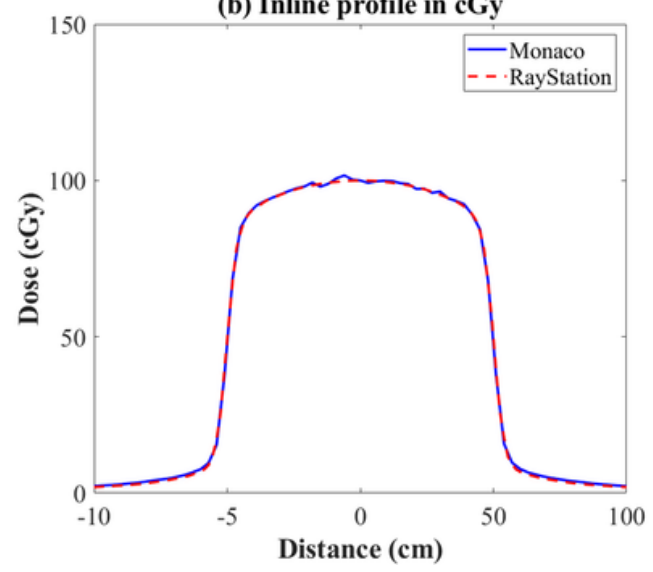

(c) Crossline profile in cGy

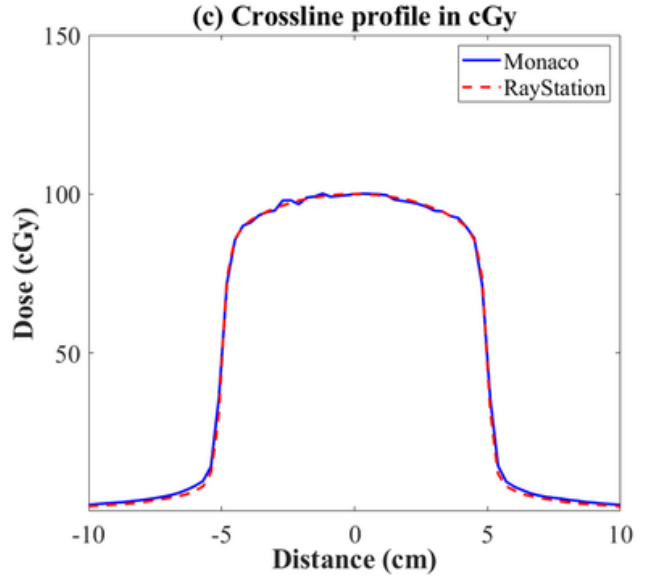

Figure 2

An example of visual inspection from a simple single field plan with a $10 \times 10 \mathrm{~cm} 2$ field size and a $10 \mathrm{~cm}$ depth. (a) A PDD curve, (b) inline and (c) crossline profiles. The blue solid and red dotted lines indicate Monaco and RayStation simple single field plans, respectively. 


\section{(a) The quality of simple single field plans in $2 \% / 2 \mathrm{~mm}$ criteria}

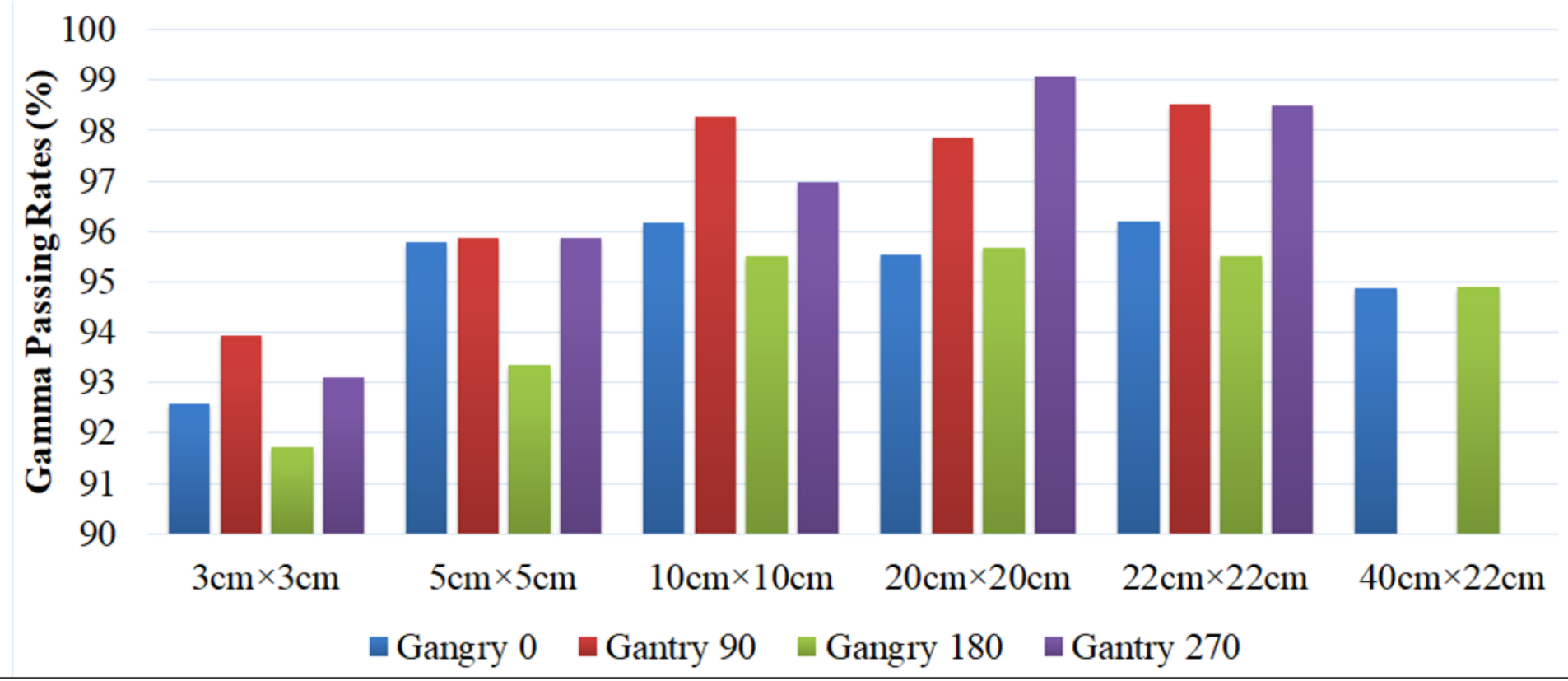

(b) The quality of simple single field plans in $3 \% / 3 \mathrm{~mm}$ criteria

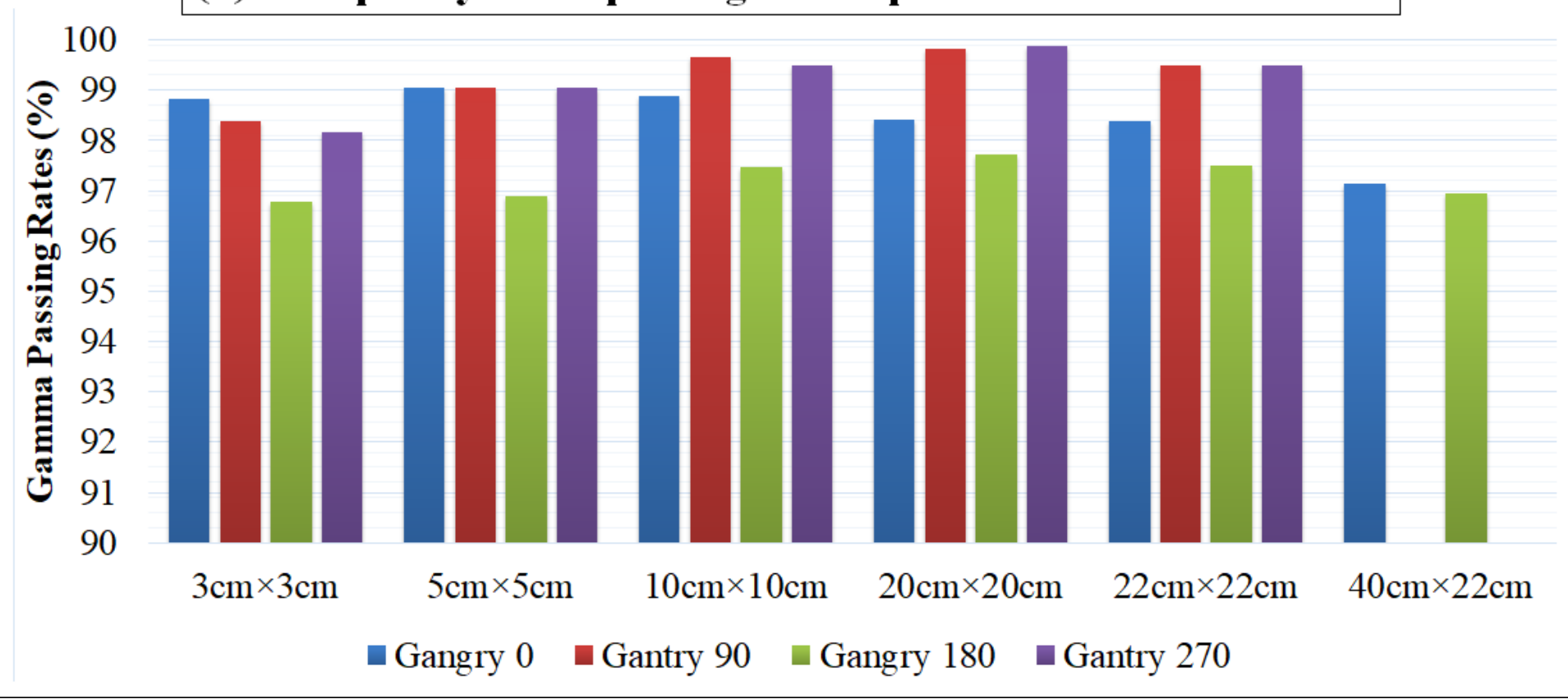

Figure 3

The quality of RayStation simple single field plans measured by comparing 3D doses with corresponding Monaco simple single field plans using the in-house gamma analysis software. (a) The quality of RayStation simple single field plans in $2 \% / 2 \mathrm{~mm}$ gamma criteria and (b) the quality of RayStation simple single field plans in $3 \% / 3 \mathrm{~mm}$ gamma criteria. 

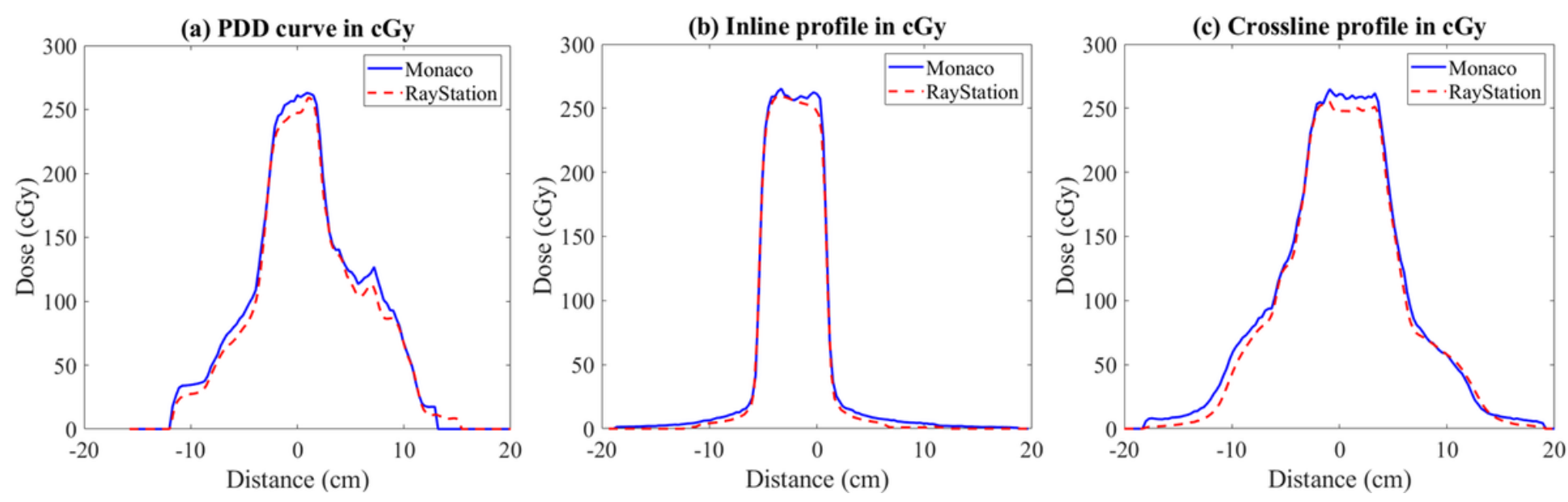

Figure 4

An example of visual inspection from a 7-field IMRT plan (250 cGy in a fraction). (a) Longitudinal, (b) inline and (c) crossline profiles at the center of axis. The blue solid and red dotted lines indicate Monaco and RayStation 7-field IMRT plans, respectively. 
(a) Monaco plan dose

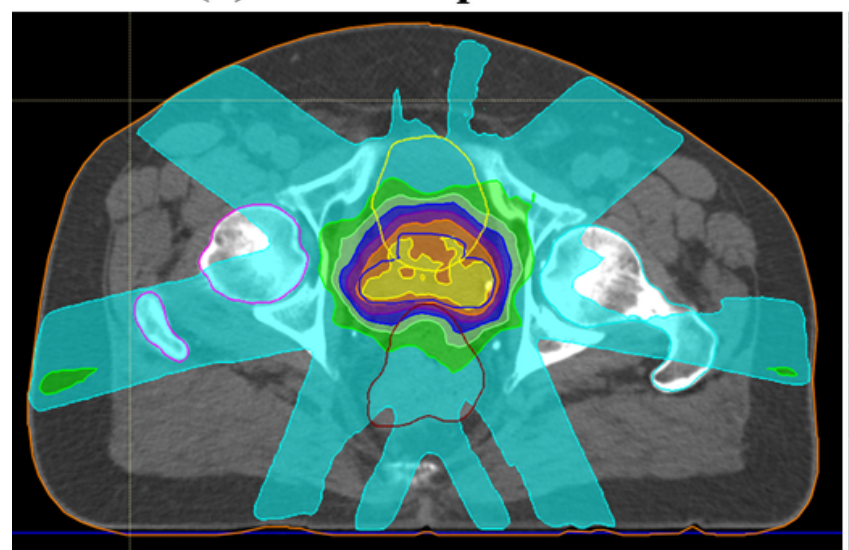

(b) RayStation plan dose
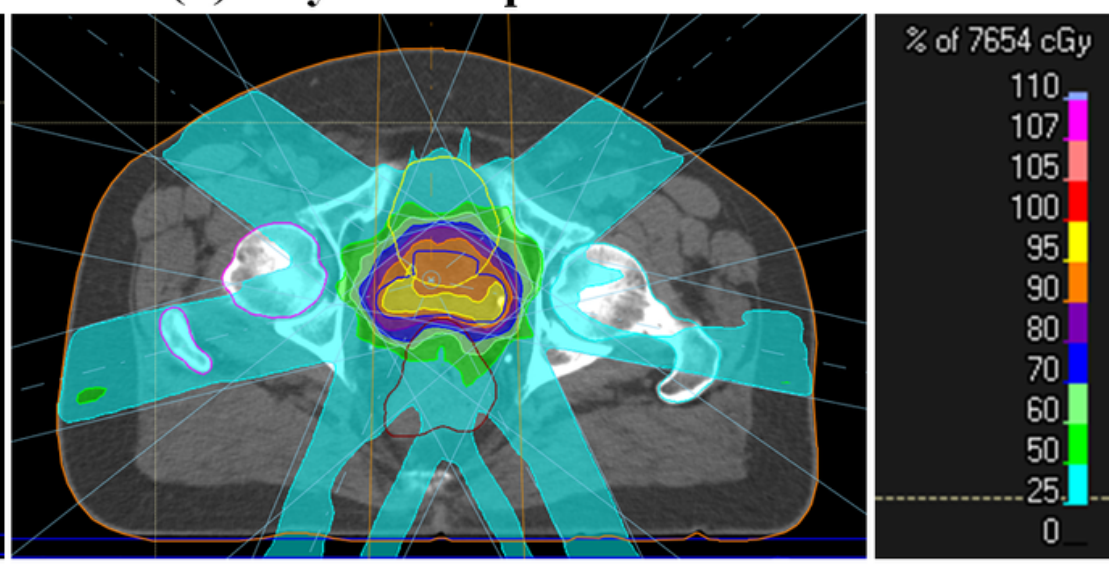

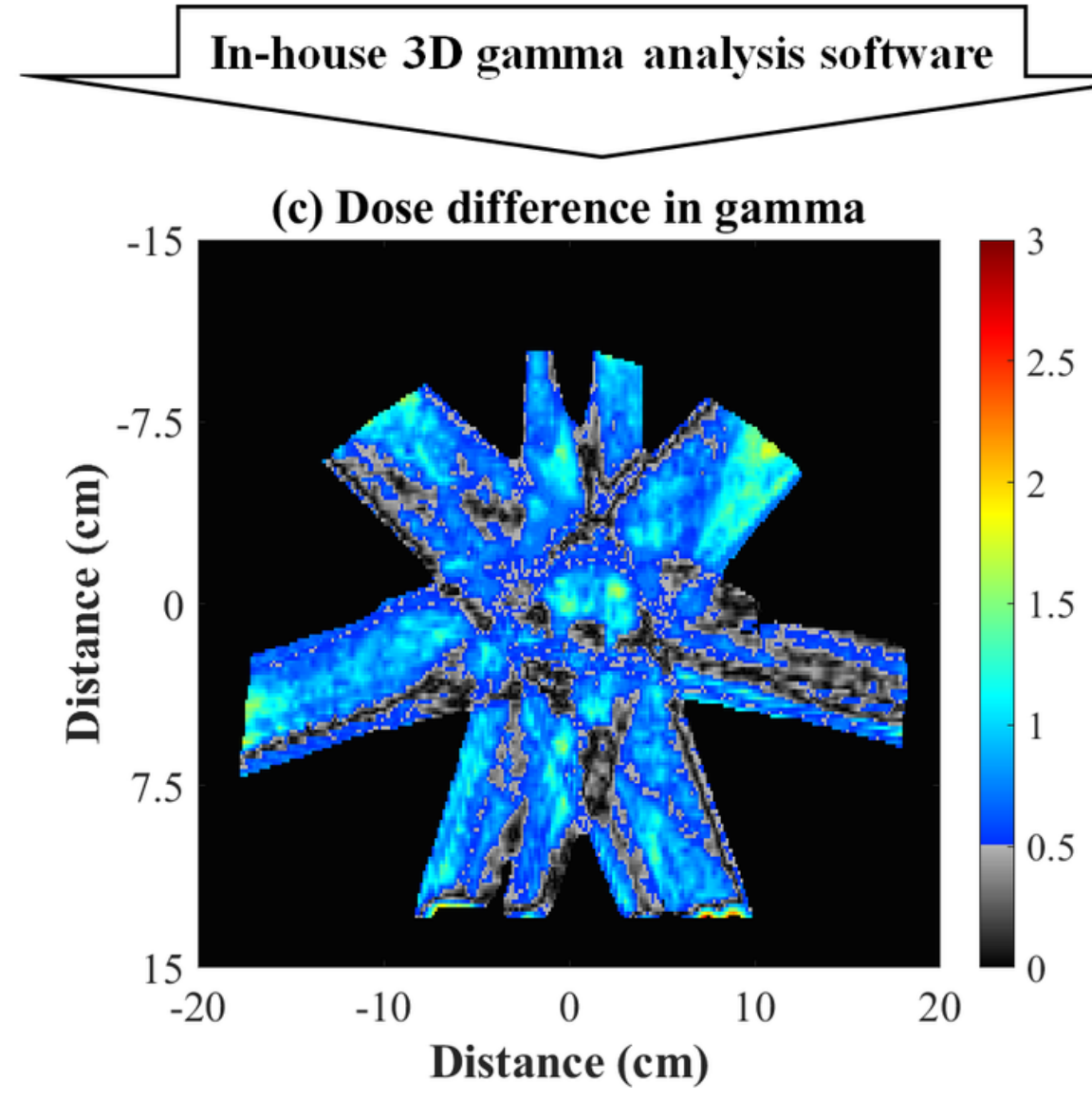

Figure 5

Monaco and RayStation 7-field IMRT plans and doses (7000 cGy in 28 fractions) displayed on RayStation. (a) Monaco plan dose calculated using a Monte Carlo algorithm, (b) RayStation plan dose calculated using a Collapsed Cone algorithm and (c) the dose difference of Monaco and RayStation plans analyzed in the in-house 3D gamma analysis software. The color bar indicates dose difference in gamma (\%). 